ARTIGO ORIGINAL / ORIGINAL ARTICLE

\title{
Métodos e aspectos operacionais de um estudo epidemiológico e de avaliação da Rede Cegonha
}

\author{
Methods and operational aspects of an epidemiological study and \\ evaluation of Rede Cegonha
}

\begin{abstract}
Antonio Fernando Boing' (D), Josimari Telino de Lacerda' (D), Alexandra Crispim Boing' (D), Maria Cristina Marino Calvo' (D), Suelen Saraiva" (D), Yaná Tamara Tomasill' (D), Sonia Natal' (D), Kátia Jakovljevic Pudla' (D), Ana Lúcia Danielewiczv (D), Vilson Rodrigues da Silva' (D), Celia Adriana Nicolotti' (D), Patricia Maria de Oliveira Machadovi (D), Tatiane Baratieri' (D)
\end{abstract}

RESUMO: Objetivo: Descrever aspectos metodológicos e operacionais de um estudo epidemiológico e de avaliação da Rede Cegonha em Santa Catarina, Brasil. Métodos: A pesquisa realizada em 2019 foi composta de dois subestudos. No primeiro, cujo desenho foi epidemiológico e que analisou os cuidados recebidos no prénatal, parto e puerpério imediato por gestantes, puérperas e crianças no Sistema Único de Saúde (SUS), são descritos os instrumentos de coleta dos dados e a organização da etapa de campo do estudo. O segundo foi uma avaliação normativa da gestão municipal na atenção ao pré-natal e puerpério no âmbito da Rede Cegonha. Iniciou-se com um estudo de avaliabilidade, seguido da avaliação propriamente dita. São descritas as diferentes estratégias metodológicas adotadas, com o envolvimento de stakeholders e especialistas. Resultados: A taxa de participação das mulheres entrevistadas no subestudo epidemiológico foi de $97,7 \%$. Mulheres residentes em $82,7 \%$ dos municípios catarinenses foram entrevistadas. A amostra foi semelhante ao registrado no Sistema de Informações sobre Nascidos Vivos (SINASC) para o mesmo período, e o perfil da subamostra entrevistada após seis meses foi semelhante ao da amostra global. O estudo avaliativo desenvolveu e aplicou modelo com 32 indicadores, que permitiu analisar a gestão sob dois aspectos: político-organizacional e tático-operacional. Contou com a adesão de 204 municípios catarinenses $(69,1 \%)$, avaliados segundo o porte populacional. Conclusão: A disponibilização de procedimentos metodológicos que possibilitem a articulação de estudos da epidemiologia e da avaliação em saúde permite gerar informações mais precisas e completas para contribuir para o delineamento e a avaliação de políticas, programas e ações de saúde do SUS.

Palavras-chave: Epidemiologia. Avaliação em saúde. Pesquisa sobre serviços de saúde. Métodos.

\footnotetext{
'Programa de Pós-Graduação em Saúde Coletiva, Universidade Federal de Santa Catarina - Florianópolis (SC), Brasil. "Instituto Federal de Santa Catarina - Florianópolis (SC), Brasil. "'Universidade Federal da Fronteira Sul - Chapecó (SC), Brasil. "Universidade Federal de Santa Catarina - Curitibanos (SC), Brasil.

vPrograma de Pós-Graduação em Ciências da Reabilitação, Universidade Federal de Santa Catarina - Araranguá (SC), Brasil. vGraduação em Medicina, Universidade Federal da Integração Latino-Americana - Foz do Iguaçu (PR), Brasil.

Autor correspondente: Antonio Fernando Boing. Departamento de Saúde Pública, Universidade Federal de Santa Catarina. Campus Universitário Reitor João David Ferreira Lima, s/n, Trindade, CEP: 88040-900, Florianópolis, SC, Brasil. E-mail: antonio.boing@ufsc.br Conflito de interesses: nada a declarar - Fonte de financiamento: Fundação de Amparo à Pesquisa do Estado de Santa Catarina (FAPESC), sob o termo de outorga n² 2017TR1364.
} 
ABSTRACT: Objective: To describe the methodological and operational aspects of an epidemiological and an evaluation of Rede Cegonha in Santa Catarina, Brazil. Methods: The research carried out in 2019 was composed of two sub-studies. Regarding the first, whose design was epidemiological and analyzed prenatal, delivery and immediate puerperium care addressed to pregnant women, puerperal women and children assisted at SUS, the instruments used for data collection and the organization of the field of the study are described. The other sub-study was a normative assessment of municipal management in prenatal and postpartum care within the scope of Rede Cegonha. It began with an evaluability assessment followed by the assessment itself. The different methodological strategies adopted are described, with the involvement of stakeholders and experts. Results: The response rate of the epidemiological sub-study was $97.7 \%$. Women residing in $82.7 \%$ of Santa Catarina's municipalities were interviewed. The sample was similar to that registered in SINASC for the same period, and the characteristics of the sub-sample interviewed after six months was similar to the global sample. The evaluation study improved and applied a model with 32 indicators that allowed to analyze the municipalities considering the political-organizational and tactical-operational aspects. Two hundred and four municipalities answered the questionnaire (69.1\%); they were evaluated according to their respective population size. Conclusion: The availability of methodological procedures of studies that articulate epidemiological and evaluation methods allows generating more accurate and complete information and contribute with the design and evaluation of health policies, programs and actions.

Keywords: Epidemiology. Health evaluation. Health services research. Methods.

\section{INTRODUÇÃO}

Melhorar a saúde e evitar a mortalidade da mulher e do bebê são dois dos principais objetivos mundiais e promovem sucessivas discussões sobre medidas necessárias e eficazes para que sejam alcançados ${ }^{1}$. Ainda que o Brasil tenha conseguido reduzir a taxa de mortalidade na infância e a razão de mortes maternas, os valores ainda são elevados, o que tem mobilizado diferentes instâncias de governo e sociedade civil a propor políticas, programas e ações para o enfrentamento desses problemas de saúde pública ${ }^{2,3}$.

A Rede Cegonha foi instituída em 2011, no Brasil, para contribuir com a redução da morbimortalidade materna e infantil. Seus objetivos são estruturar e organizar os serviços de saúde como uma rede de atenção, apresentando quatro componentes estratégicos de ação:

- o relacionado ao pré-natal;

- o de parto e nascimento;

- o vinculado ao puerpério e à atenção à saúde integral da criança;

- o vinculado ao sistema logístico ${ }^{4}$.

Do conjunto de atividades a ser desenvolvidas para melhorar as condições de saúde de mulheres e crianças, destaca-se o alto potencial de atuação das equipes da Atenção Primária à Saúde (APS) na redução da morbimortalidade materna e infantil, por meio de um atendimento qualificado durante a gestação e o pós-parto ${ }^{5-7}$. No entanto, a despeito de estar ocorrendo aumento da cobertura da assistência pré-natal e do número de 
consultas por gestantes no Brasil ${ }^{3,8,9}$, indicadores como os de incidência de sífilis congênita e de hipertensão arterial sistêmica mantêm-se elevados, levando ao questionamento da qualidade dessa atenção ${ }^{10,11}$.

A elaboração e a implantação de instrumentos de avaliação das ações desenvolvidas pelos municípios no cuidado pré-natal e no pós-parto podem colaborar para a identificação de aspectos frágeis e fortes na APS. Da mesma forma, dados empíricos de amostras probabilísticas sobre os cuidados recebidos pela gestante durante o pré-natal e no pós-parto podem subsidiar a definição de ações e políticas públicas para a melhoria da qualidade da assistência. Investigações com tais desenhos são escassos no Brasil, particularmente estudos epidemiológicos articulados a outros de avaliação, com um conjunto amplo de análises e indicadores dos serviços de saúde sobre o cuidado à mulher e à criança. Assim, apresentar e discutir percursos metodológicos, desafios e soluções de estudos que articulem ferramentas e conceitos da epidemiologia e da avaliação em saúde nas investigações sobre saúde da mulher e da criança pode contribuir para a disseminação de tais pesquisas na área.

Este artigo propõe-se a descrever os aspectos metodológicos de um estudo que associou ferramentas da epidemiologia e da avaliação em saúde para realizar um diagnóstico da atenção ao pré-natal e pós-parto no estado de Santa Catarina, com dados primários de puérperas e uma avaliação da gestão municipal de dois componentes da Rede Cegonha.

\section{MÉTODOS}

O trabalho desdobrou-se em dois subestudos complementares, aliando métodos da epidemiologia e da avaliação em saúde. O primeiro coletou dados primários de puérperas de Santa Catarina, com o objetivo de identificar o recebimento de cuidados na atenção básica durante o pré-natal e o pós-parto. O segundo construiu e aplicou um modelo avaliativo da gestão municipal na atenção ao pré-natal e pós-parto. Os métodos distintos de cada investigação serão apresentados separadamente.

\section{SUBESTUDO 1: COLETA DE DADOS JUNTO ÀS PUÉRPERAS}

\section{População de referência para o estudo}

A população de estudo compôs-se de puérperas que:

- realizaram o parto no Sistema Único de Saúde (SUS) em Santa Catarina;

- residiram em Santa Catarina durante toda a gestação;

- realizaram todas as consultas de pré-natal no SUS ou não realizaram qualquer consulta de pré-natal;

- tiveram filhos nascidos vivos ou natimortos que nasceram com mais de $500 \mathrm{~g}$ e ao menos 22 semanas de gestação nas 48 horas anteriores à entrevista. 
Foram incluídos na investigação todos os hospitais de Santa Catarina que, em 2016, realizaram ao menos 500 partos pelo SUS, o que resultou no total de 31 hospitais distribuídos em 30 diferentes municípios. Esse conjunto de instituições correspondeu a 86,2\% de todos os nascimentos no estado financiados pelo SUS em 2016. O cálculo amostral foi realizado no software Stata 15. Com nível de confiança de $95 \%$, população de 50 mil, margem de erro de 1,6 ponto percentual, prevalência estimada de 50 e $5 \%$ para recompor perdas, a amostra incluiu 3.665 mulheres. O número de entrevistas realizadas em cada hospital seguiu a distribuição proporcional de nascimentos observada em 2016.

\section{Instrumento de coleta de dados}

O instrumento de coleta de dados foi construído com base em reuniões periódicas que contaram com a participação de docentes e pós-graduandos. Como a pesquisa tinha um forte componente de avaliação dos serviços de saúde do estado, as discussões para a construção do questionário também envolveram diferentes profissionais da Secretaria de Estado da Saúde (SES).

A versão final do questionário foi composta de 365 questões, algumas oriundas de instrumentos fechados e validados trazidos de outras pesquisas com objetivos semelhantes e outras desenhadas pelos pesquisadores conforme os objetivos do projeto. Esse instrumento estava dividido em doze blocos:

- Critérios de Inclusão;

- Informações Iniciais;

- Identificação;

- Fatores Comportamentais;

- Assistência Pré-Natal;

- Satisfação/Discriminação no Pré-Natal;

- Parto/Nascimento;

- Satisfação/Discriminação no Parto;

- Ambiente de Moradia;

- Socioeconômico;

- Demográfico;

- Caderneta de Pré-Natal.

O questionário completo foi testado em 35 pessoas para verificar o entendimento de cada questão e a estruturação do instrumento como um todo.

O manual do entrevistador apresentava informações detalhadas sobre o que se buscava medir em cada pergunta, o comportamento que o pesquisador deveria assumir no questionamento, as dúvidas possíveis que as entrevistadas poderiam manifestar e as explicações a ser repassadas. Também contemplava questões éticas e instruções sobre o comportamento a ser adotado no trato com a equipe dos hospitais, as puérperas e os acompanhantes. Os entrevistadores foram capacitados para a aplicação da pesquisa no ambiente hospitalar em intensa dinâmica de treinamento e de forma descentralizada no estado. 


\section{Equipe de entrevistadores}

Foram selecionados 35 entrevistadores com, no mínimo, ensino superior incompleto na área da saúde. Tal estratégia permitiu a seleção de pesquisadores familiarizados com o tema e que residiam próximo aos hospitais.

\section{Coleta dos dados e controle de consistência}

O questionário foi programado na plataforma do Research Electronic Data Capture (REDCap) Brasil (https: / / www.redcapbrasil.com.br/) instalada em tablets. Ao fim de cada dia, os entrevistadores conectavam-se a uma rede wi-fi e transmitiam os dados ao servidor do estudo, abrigado na Universidade Federal de Santa Catarina. Diariamente, a coordenação da pesquisa testava a consistência e a qualidade dos dados por meio de análise descritiva. Os resultados eram repassados aos coordenadores regionais, que os transmitiam aos entrevistadores e reforçavam as orientações pertinentes. Com isso, uma nova visita ao hospital permitia a correção das inconsistências no prazo de horas ou poucos dias.

As informações sobre as puérperas foram extraídas de suas cadernetas de gestante (quando disponíveis) e dos prontuários hospitalares. O acesso às três fontes permitiu obter-se a amplitude necessária de dados para o estudo e, no caso de repetição de dados em diferentes fontes, possibilitou comparar as informações autorreferidas com as registradas nos documentos. A coleta de dados ocorreu entre os meses de janeiro e agosto de 2019.

\section{Logística de campo}

O instrumento de coleta foi submetido a estudo piloto em quatro hospitais participantes do estudo, com o equivalente a $5 \%$ da amostra. Essa etapa permitiu verificar a necessidade de ajustes no questionário, possibilitou a mensuração do tempo de aplicação da pesquisa que variou de 40 a 60 minutos - e permitiu testar a dinâmica do registro e da transmissão dos dados pelo REDCap até o servidor central.

\section{Controle de qualidade}

O controle de qualidade dos dados foi realizado semanalmente em amostra aleatória de $10 \%$ das entrevistadas. As mulheres sorteadas eram contatadas por telefone pelos supervisores regionais, que aplicavam questionário reduzido com oito perguntas cujas respostas não seriam passíveis de modificação entre os dois momentos de entrevista. Buscaram-se, com a prática, identificar eventuais fraudes (criação de entrevistas inexistentes por um entrevistador) e medir a reprodutibilidade das medidas. As perguntas incluídas no controle de qualidade referiam-se a tipo de parto, número de gestações anteriores, realização de consulta 
odontológica no pré-natal, fumo durante o período gestacional, visita à maternidade previamente ao parto, escolaridade máxima, uso de sulfato ferroso durante a gestação e realização de vacina durante o pré-natal.

\section{Segunda entrevista após seis meses}

Uma segunda entrevista foi realizada seis meses após o parto, com o objetivo de verificar o atendimento recebido pela mulher e pela criança nesse período, com foco no puerpério. Para isso, 975 mulheres que participaram da primeira coleta foram entrevistadas novamente por meio telefônico. Nessa etapa, o instrumento de coleta foi um questionário estruturado, com 41 perguntas divididas em oito blocos: identificação, situação da criança, cuidado à mulher e à criança no puerpério, aleitamento materno e alimentação da criança, acompanhamento da equipe de saúde, planejamento reprodutivo e educação sexual, comportamentos e discriminação no puerpério. Essa fase seguiu a mesma dinâmica de programação de dados utilizada anteriormente, e sete entrevistadores participaram da coleta. Antecipando essa etapa, a equipe de pesquisa armazenou a maior diversidade de contatos telefônicos durante a primeira onda, com o intuito de minimizar as perdas.

\section{SUBESTUDO 2: AVALIAÇÃO DA ATENÇÃO PRIMÁRIA NOS MUNICÍPIOS}

Trata-se de uma avaliação normativa realizada em duas etapas, tendo como objeto de estudo a gestão municipal na atenção ao pré-natal e puerpério no âmbito da Rede Cegonha em Santa Catarina.

A primeira etapa consistiu em um estudo de avaliabilidade ${ }^{12-14}$, que adotou como estratégias metodológicas análise documental, reuniões, seminários e oficinas de consenso envolvendo stakeholders e especialistas em avaliação e atenção primária.

A análise documental incluiu a leitura de materiais técnicos que orientam a implantação da Rede Cegonha em nível federal disponíveis no site do Ministério da Saúde (Quadro 1). Procedeu-se à análise crítica dos materiais, buscando ampliar a compreensão sobre o objeto, com ênfase nos aspectos que compõem a atenção materna e infantil na atenção básica do âmbito municipal dos serviços de saúde, seus objetivos, metas, atividades, produtos, resultados, impactos e o papel da gestão municipal.

Foram realizadas duas reuniões com a participação de profissionais da APS (médicos e enfermeiros) e representantes das gestões estadual e municipal de saúde $(\mathrm{n}=10)$ para a discussão dos objetivos, atividades, produtos e resultados esperados, identificados na análise documental.

Com base nos resultados dessa análise e nas reuniões, definiram-se perguntas avaliativas e elaboraram-se o Modelo Teórico (MT) e o Modelo Lógico (ML), que consistem na representação esquemática do objeto avaliativo, ampliando a compreensão da natureza do problema, da intervenção e da relação do objeto com o contexto. As propostas 
Quadro 1. Referências técnicas consultadas para a análise documental no estudo avaliativo da Rede Cegonha. Santa Catarina, 2019.

\begin{tabular}{|c|c|}
\hline Referência Técnica & Conteúdo Principal \\
\hline $\begin{array}{l}\text { Portaria SAS/MS } \\
650 / 2011\end{array}$ & $\begin{array}{l}\text { Regulamenta a elaboração dos planos de ação regional e municipal } \\
\text { da Rede Cegonha para a execução das fases de implementação da rede, } \\
\text { bem como o repasse de recursos, avaliação e monitoramento. }\end{array}$ \\
\hline $\begin{array}{l}\text { Portaria da Rede Cegonha } \\
\text { GM/MS 1.459/2011 }\end{array}$ & Institucionalização da Rede Cegonha no âmbito do SUS. \\
\hline $\begin{array}{l}\text { Portaria de Redes GM/MS } \\
4.279 / 2010\end{array}$ & $\begin{array}{l}\text { Estabelece diretrizes para a organização da Rede de } \\
\text { Atenção à Saúde no âmbito do SUS. }\end{array}$ \\
\hline $\begin{array}{l}\text { Política Nacional de } \\
\text { Atenção Integral à Saúde } \\
\text { das Mulheres, MS }\end{array}$ & $\begin{array}{c}\text { Estabelece princípios e diretrizes de qualidade e humanização } \\
\text { para a saúde das mulheres, direito sexual e reprodutivo, abrangendo } \\
\text { todos os programas anteriores. }\end{array}$ \\
\hline $\begin{array}{l}\text { Política Nacional de } \\
\text { Atenção Integral à Saúde } \\
\text { da Criança Portaria GM/ } \\
\text { MS } 1.130 / 2015\end{array}$ & $\begin{array}{c}\text { Estabelece os princípios e diretrizes para a promoção e proteção da } \\
\text { saúde da criança, mediante atenção e cuidados integrais desde a } \\
\text { gestação, visando à redução da morbimortalidade e condições } \\
\text { dignas de existência e pleno desenvolvimento. }\end{array}$ \\
\hline $\begin{array}{l}\text { Política Nacional de } \\
\text { Atenção Básica, MS }\end{array}$ & $\begin{array}{l}\text { Estabelece a Estratégia Saúde da Família como modelo prioritário para } \\
\text { organização da Atenção Primária à Saúde, responsabilidade sanitária das } \\
\text { equipes e municípios para assistência integral à saúde de mulheres e crianças. }\end{array}$ \\
\hline $\begin{array}{l}\text { Portaria de Consolidação } \\
\text { n }^{\circ} 02 / 2017 \text {, MS }\end{array}$ & ăo das normas sobre políticas nacionais do SUS. \\
\hline
\end{tabular}

SAS: Secretaria de Assistência à Saúde; MS: Ministério da Saúde; GM: Gabinete do Ministério; SUS: Sistema Único de Saúde.

preliminares do MT e do ML foram objeto de discussão e ajustes em um seminário acadêmico promovido por um núcleo de pesquisa em avaliação em saúde, com a participação de estudiosos nos temas da atenção ao pré-natal, parto e nascimento e puerpério e da gestão da atenção primária.

Conferências de consenso ${ }^{15}$ foram realizadas com especialistas identificados pela sua atuação profissional e acadêmica nas áreas de epidemiologia, planejamento e avaliação em saúde. Também foram incluídos os stakeholders (interessados na avaliação) ligados à gestão da atenção básica no SUS em nível estadual e municipal ${ }^{15}$. Para estabelecer o consenso, os participantes $(n=14)$ receberam em mídia eletrônica a proposta preliminar do modelo avaliativo com um prazo de 15 dias para análise, podendo responder se concordavam totalmente, parcialmente ou discordavam de cada um dos componentes do modelo, justificando a sua escolha. As sugestões e alterações do MT e ML propostas foram apresentadas e debatidas em duas oficinas presenciais, com duração de quatro horas cada. Na sequência, duas novas oficinas presenciais foram realizadas para a apreciação da Matriz de Análise e Julgamento (MAJ), com o mesmo tempo de duração e menor número de participantes ( $\mathrm{n}=10$ ).

O conteúdo das oficinas presenciais foi descrito e sistematizado por três pesquisadoras que organizaram uma nova proposta pautada pelas decisões do grupo. O modelo avaliativo reestruturado foi reenviado aos participantes para revisão e validação, obtendo-se o consenso final da proposta e sugestões de estratégias para sua aplicação. 
A segunda etapa de avaliação consistiu na coleta e análise de dados da gestão municipal, com base nos produtos obtidos no estudo de avaliabilidade. Foram identificadas as fontes de evidência dos elementos da MAJ, inicialmente pela análise da disponibilidade de informações em bancos de dados de pesquisa e em páginas eletrônicas oficiais. Observou-se que a grande maioria das informações estava disponível em fontes de dados secundários para o ano de 2017, mas que havia necessidade de complementação com a coleta de dados primários dos gestores municipais.

Procedeu-se à elaboração e validação do questionário encaminhado a todos os gestores municipais de saúde de Santa Catarina $(n=295)$ pela plataforma de pesquisa on-line Survey Monkey. Em parceria com a SES, foram definidas e aplicadas estratégias de sensibilização para envio e monitoramento do preenchimento do formulário pelos gestores municipais. Em acréscimo, foram adotadas medidas de reforço pelos pesquisadores direcionadas aos gestores municipais não respondentes ou que iniciaram, mas não concluíram, o envio do questionário.

Um protocolo com a identificação detalhada dos componentes da MAJ foi elaborado em planilha eletrônica para orientar a organização e a análise dos dados segundo dimensões, subdimensões, indicadores, medidas, variáreis, fonte de evidência, base de cálculo e parâmetros de julgamento.

Para a análise, os municípios foram agrupados, segundo o porte populacional, em seis estratos (por número de habitantes): até 3 mil; de 3.001 a 5.999; de 6.000 a 9.999; de 10.000 a 19.999; de 20.000 a 49.999; e igual ou superior a 50 mil.

Para a emissão de juízo de valor orientado pelo somatório dos elementos avaliativos, assumiu-se como adequado o alcance de pelo menos $75 \%$ do limite máximo possível em cada um dos componentes da matriz avaliativa, como inadequado o alcance de pelo menos 50\% e os demais como parcialmente adequados.

\section{ASPECTOS ÉTICOS DO ESTUDO}

A pesquisa obteve aprovação do Comitê de Ética em Pesquisa com Seres Humanos (CEPSH) da UFSC. Foram seguidos todos os preceitos éticos da Resolução do Conselho Nacional de Saúde n ${ }^{\circ}$ 510/2016. Todas as entrevistadoras assinaram um Termo de Sigilo das Informações e as entrevistadas um Termo de Consentimento Livre e Esclarecido.

\section{RESULTADOS}

\section{SUBESTUDO 1}

Participaram do estudo 3.580 mulheres, entrevistadas nos 31 hospitais participantes do estudo (Figura 1A). Assim, a taxa de recusa foi de 2,3\% $(\mathrm{n}=85)$ da amostra inicialmente planejada. A taxa de resposta foi superior a $83 \%$ em todos os hospitais - em 25 deles foi de $100 \%$ e em outros três variou entre 98 e $99 \%$. Os questionários construídos no estudo e aplicados após 48 horas e após seis meses do parto estão disponibilizados no Material Suplementar. 
A

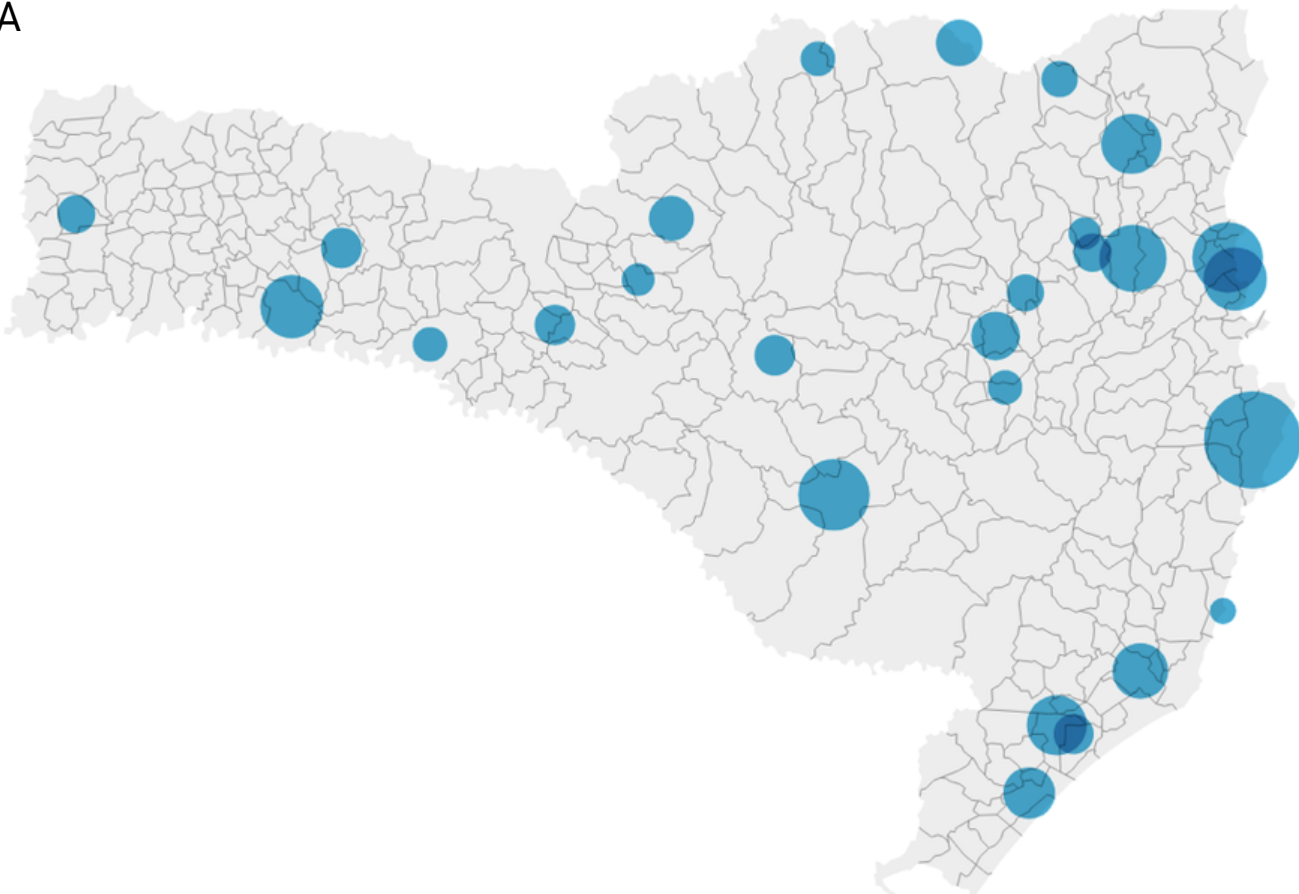

B

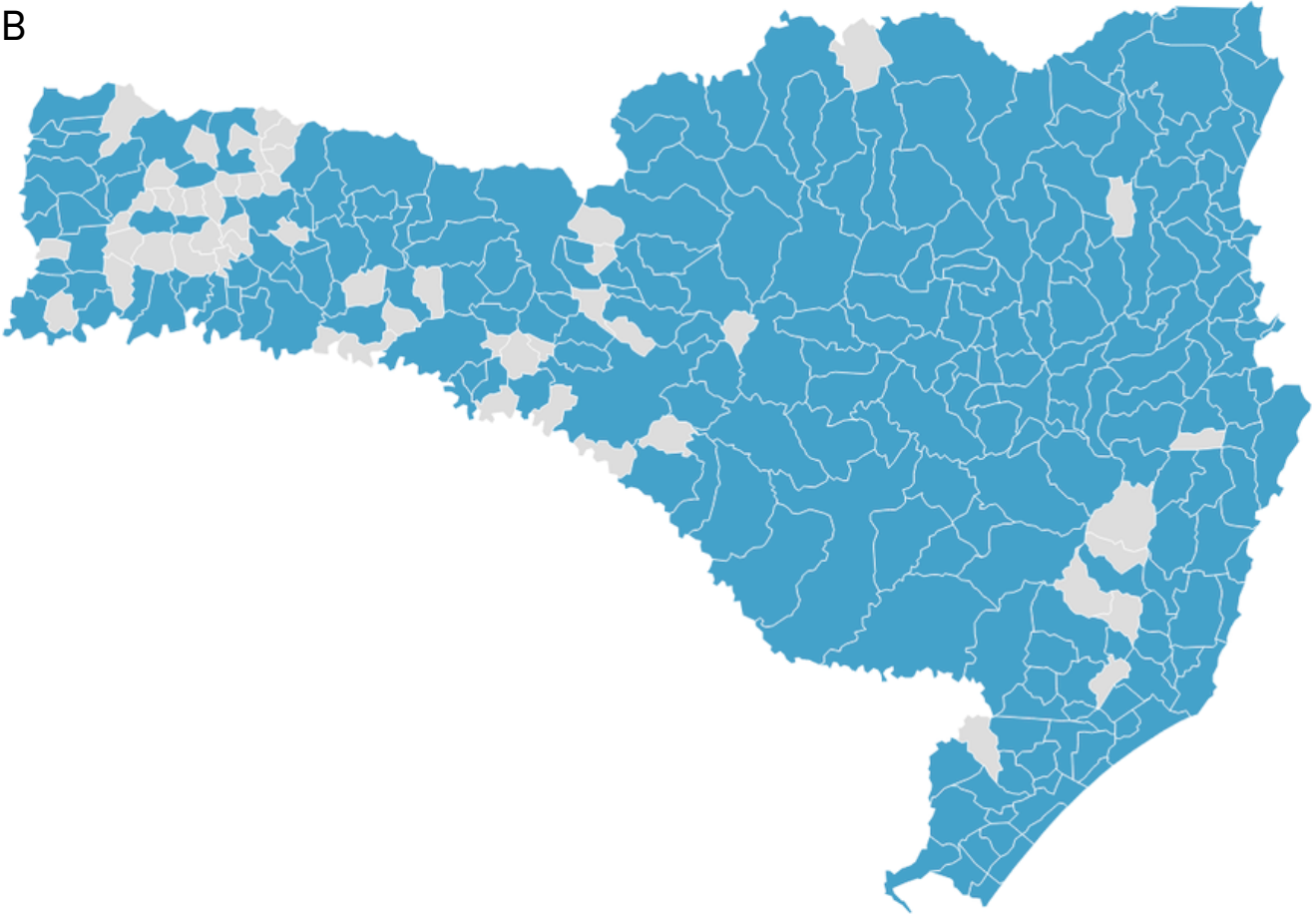

Figura 1. (A) Distribuição espacial dos 31 hospitais participantes do estudo de acordo com a proporção de nascimentos registrados e (B) municípios em azul com ao menos uma gestante residente entrevistada. Santa Catarina, 2019. 
Mulheres residentes em 244 municípios catarinenses, do total de 295 que compõem o estado, fizeram parte da amostra (Figura 1B). Ao se compararem os dados do presente estudo com os do Sistema de Informações sobre Nascidos Vivos (SINASC) de 2019, referentes aos mesmos hospitais, observa-se a similaridade dos valores de sexo do recém-nascido, idade e escolaridade da puérpera, número de consultas de pré-natal e tipo de parto (Tabela 1). As pequenas variações observadas podem estar relacionadas ao fato de os dados do SINASC contemplarem mulheres que realizaram o pré-natal em uma alternância público-privada ou exclusivamente no setor privado, mesmo que o parto tenha ocorrido no SUS.

Não foi identificada fraude nas entrevistas e todas as variáveis do controle de qualidade mostraram concordância boa ou quase perfeita; seis das oito variáveis analisadas apresentaram Kappa de Cohen maior que 0,68. A análise das características das mulheres entrevistadas seis meses após o parto também apresentou correspondência com a amostra global do estudo entrevistada nos hospitais (Tabela 1).

Tabela 1. Comparação entre características das puérperas observadas nos registros do SINASC para os 31 hospitais participantes da presente pesquisa e obtidas na amostra do estudo. Santa Catarina, 2019.

\begin{tabular}{|c|c|c|c|}
\hline & $\begin{array}{c}\text { Amostra do presente } \\
\text { estudo entrevistada } \\
\text { no hospital }\end{array}$ & $\begin{array}{c}\text { Dados } \\
\text { do SINASC* }\end{array}$ & $\begin{array}{l}\text { Amostra do presente } \\
\text { estudo entrevistada por } \\
\text { telefone no seguimento }\end{array}$ \\
\hline \multicolumn{4}{|c|}{ Sexo do recém-nascido } \\
\hline Feminino & 49,5 & 49,0 & 48,1 \\
\hline Masculino & 50,5 & 51,0 & 51,9 \\
\hline \multicolumn{4}{|c|}{ Faixa etária da puérpera (idade em anos) } \\
\hline$>=19$ & 13,4 & 12,4 & 13,8 \\
\hline $20-34$ & 72,3 & 72,3 & 71,5 \\
\hline$>=35$ & 14,3 & 15,3 & 14,7 \\
\hline \multicolumn{4}{|c|}{ Anos de estudo da puérpera } \\
\hline$<8$ anos & 12,8 & 14,8 & 15,3 \\
\hline$>=8$ anos & 87,2 & 85,2 & 84,7 \\
\hline \multicolumn{4}{|c|}{ Número de consultas de pré-natal } \\
\hline$<=3$ & 3,2 & 4,1 & 2,5 \\
\hline $4-6$ & 17,6 & 17,8 & 18,6 \\
\hline$>=7$ & 79,3 & 78,0 & 78,9 \\
\hline \multicolumn{4}{|l|}{ Tipo de parto } \\
\hline Vaginal & 57,2 & 52,2 & 58,7 \\
\hline Cesárea & 42,8 & 47,8 & 41,3 \\
\hline
\end{tabular}

SINASC: Sistema de Informações sobre Nascidos Vivos. 


\section{SUBESTUDO 2}

As estratégias adotadas no subestudo 2 permitiram uma compreensão abrangente do objeto - do envolvimento de interessados na avaliação, da definição do plano avaliativo e de sua execução. Obteve-se a descrição das responsabilidades da gestão municipal na atenção ao pré-natal e puerpério no âmbito da Rede Cegonha, com identificação de metas, objetivos, recursos necessários, atividades pretendidas, resultados esperados e relações causais, sintetizados em diagramas dos ML e MT, além de consenso quanto aos elementos da avaliação.

O modelo avaliativo elaborado com base no referencial de Redes e da Atenção Básica considerou o contexto, as políticas de saúde, as legislações e normas relacionadas ao tema, sob diferentes pontos de vista e à luz de conhecimentos compartilhados. Permitiu analisar a gestão sob dois aspectos: político-organizacional e tático-operacional. O primeiro engloba as condições ofertadas pela gestão municipal para a garantia das ações de assistência às gestantes, puérperas e crianças no pré-natal e puerpério na Atenção Primária à Saúde. O segundo caracteriza-se pela organização da assistência e pelas ações / atividades desenvolvidas pelos profissionais da APS para o cuidado a gestantes, puérperas e crianças.

A MAJ foi composta de duas dimensões, seis subdimensões, 32 indicadores e 90 medidas e requer 94 variáveis para sua aplicação. A maioria das informações $(57,4 \%)$ está disponível no banco de dados do ciclo 3 da avaliação externa do Programa Nacional de Melhoria do Acesso e da Qualidade da Atenção Básica (PMAQ_AB) e em bases de dados oficiais de acesso público ou disponibilizadas mediante solicitação formal aos órgãos competentes. A síntese do modelo avaliativo, com as fontes de evidências utilizadas para cada um dos indicadores, e as questões formuladas aos gestores municipais em plataforma eletrônica estão disponibilizadas no Material Suplementar.

A metodologia de coleta de dados primários precisou ser ajustada ao longo do estudo. No primeiro momento, as estratégias de sensibilização e parceria com a SES possibilitaram taxa de resposta inferior a $30 \%$. Com a redefinição de estratégias e responsabilidades e a ampliação do período de coleta para seis meses, essa taxa subiu para $69,1 \%(\mathrm{n}=204)$.

Os 204 municípios que participaram da pesquisa representam as diferentes regiões do estado, sendo: 38 (18,6\%) de porte populacional de até 3 mil habitantes; igual número encontrando-se no estrato entre 3.001 e 5.999 habitantes; $36(17,6 \%)$ no estrato de 6.000 a 9.999 habitantes; $41(20,1 \%)$ no de 10.000 a 19.999 habitantes; 25 (12,3\%) no de 20.000 a 49.999; e $26(12,7 \%)$ no de população igual ou superior a 50 mil habitantes.

\section{DISCUSSÃO}

A coleta de dados primários em ampla amostra probabilística a respeito dos cuidados recebidos no pré-natal e no pós-parto por mulheres e crianças permite que o estado disponha de visão mais ampla dos aspectos fortes e frágeis da atenção em saúde ofertada a esse público no SUS. Como consequência, espera-se o fortalecimento dos processos decisórios e do desenho de políticas e ações públicas. A definição de uma linha de base também 
permitirá aos gestores e à sociedade civil dispor de elementos para a avaliação futura de ações implementadas nesse intervalo de tempo. A articulação academia-serviços de saúde, desde a etapa de planejamento do estudo, foi essencial para que os resultados refletissem questionamentos da própria gestão e de profissionais do SUS, apresentando maior possibilidade de uso pelos serviços de saúde.

Em termos operacionais, a incorporação do REDCap à pesquisa trouxe inúmeras vantagens que merecem destaque. Como todo formulário eletrônico, permitiu a inclusão de controles que diminuíram a probabilidade de erros de digitação e de pulos automáticos, que agilizam o registro dos dados. Além disso, eliminou a etapa de digitação de questionários em papel. Por fim, a possibilidade de dispor dos dados no formato eletrônico já ao fim da entrevista, em um servidor central, conferiu maior agilidade ao controle de consistência e permitiu o treinamento continuado da equipe de entrevistadores.

Um importante passo de um estudo avaliativo é o aprofundamento do conhecimento sobre o objeto, orientado por um referencial teórico ou normativo, identificando sua dinâmica operacional, expectativa de produtos e resultados, plausibilidade e viabilidade de objetivos, de modo a direcionar a escolha dos componentes da avaliação ${ }^{13,14}$. Essas etapas, que antecedem à avaliação propriamente dita, nem sempre estão apresentadas detalhadamente nas publicações da área. O envolvimento de diferentes atores, com diversificada estratégia participativa, entendimento aprofundado sobre o objeto da pesquisa e análise do potencial de sua avaliação contribui para aumentar a factibilidade, a credibilidade e a utilidade dos resultados obtidos na avaliação propriamente dita, bem como para o uso racional dos recursos ${ }^{16,17}$.

O modelo avaliativo desenvolvido, com reduzido número de indicadores, a maioria extraído de dados secundários, amplia seu potencial de reprodutibilidade em outros contextos nacionais, permitindo a análise comparativa da conformação da Rede Cegonha. Uma limitação metodológica que requer ajuste para ampliar o potencial anteriormente descrito é a vinculação do objeto à Rede Cegonha, uma proposta de governo que pode ser desestruturada com a alternância de mandatos. Cabe ressaltar o cuidado adotado para a seleção de indicadores, orientada pelo referencial da Atenção Primária e das Redes de Atenção, demandando ajustes pontuais em poucas medidas, sem prejuízo do modelo.

\section{REFERÊNCIAS}

1. Countdown to 2030 Collaboration. Countdown to 2030: tracking progress towards universal coverage for reproductive, maternal, newborn, and child health. Lancet 2018; 391(10129): 1538-48. https:// doi.org/10.1016/s0140-6736(18)30104-1

2. Brasil. Instituto de Pesquisa Econômica Aplicada e Secretaria de Planejamento e Investimentos Estratégicos. Objetivos de Desenvolvimento do Milênio: relatório nacional de acompanhamento. Brasília: IPEA; 2014.
3. Leal MC, Szwarcwald CL, Almeida PVB, Aquino EML, Barreto ML, Barros F, et al. Saúde reprodutiva, materna, neonatal e infantil nos 30 anos do Sistema Único de Saúde (SUS). Ciênc Saúde Colet 2018; 23(6): 1915-28. https: / / doi.org/10.1590/1413-81232018236.03942018

- Brasil. Ministério da Saúde. Portaria $n^{\circ} 1.459$, de 24 de junho de 2011. Institui, no âmbito do Sistema Único de Saúde-SUS, a Rede Cegonha [Internet]. Brasil: Ministério da Saúde; 2011. Disponível em http:/ / bvsms.saude.gov. br/bvs/saudelegis/gm/2011/prt1459_24_06_2011.html 
5. Espanha. Grupo de trabajo de Guía de práctica clínica de atención em el embarazo y puerperio. Guía de práctica clínica de atención em el embarazo y puerperio. Andalucía: Ministerio de Sanidad, Servicios Sociales e Igualdad, Agencia de Evaluación de Tecnologías Sanitarias de Andalucía; 2014.

6. Viellas EF, Domingues RMSM, Dias MAB, Gama SGN, Theme Filha MM, Costa JV, et al. Assistência pré-natal no Brasil. Cad Saúde Pública 2014; 30(Supl.1): S85S100. https:// doi.org/10.1590/0102-311X00126013

7. Correa MSM, Feliciano KVO, Pedrosa EN. Souza AI. Acolhimento no cuidado à saúde da mulher no puerpério. Cad Saúde Pública 2017; 33(3): e00136215. https: / / doi.org/10.1590/0102-311x00136215

8. Mallmann MB, Boing AF, Tomasi YT, Anjos JC, Boing AC. Evolução das desigualdades socioeconômicas na realização de consultas de pré-natal entre parturientes brasileiras: análise do período 2000-2015. Epidemiol Serv Saúde 2018; 27(4): e2018022. https:// doi. org/10.5123/s1679-49742018000400014

9. Brasil. Sistema de Informações sobre Nascidos Vivos [Internet]. Brasil: Ministério da Saúde; 2020 [acesso em 12 maio 2020]. Disponível em: http: / / tabnet.datasus. gov.br/cgi/deftohtm.exe?sinasc/cnv/nvuf.def

10. Brasil. Ministério da Saúde. Secretaria de Atenção à Saúde. Departamento de Atenção Básica. Atenção ao pré-natal de baixo risco. Brasília: Ministério da Saúde; 2012.

11. Pacagnella RC, Nakamura-Pereira M, Gomes-Sponholz F, Aguiar RALP, Guerra GVQL, Diniz CSG, et al. Maternal Mortality in Brazil: Proposals and Strategies for its Reduction. Rev Bras Ginecol Obstet 2018; 40(9): 501-6. https: / / doi.org/10.1055/s-0038-1672181
12. Centers for Disease Control and Prevention (CDC). Framework for Program Evaluation in Public Health. MMWR Recomm Rep 1999; 48(11): 1-40.

13. Thurston W, Ramaliu A. Evaluability assessment of a survivors of torture program: lessons learned. Can J Program Eval 2005; 20(2): 1-25.

14. Trevisan MS, Walser TM. Evaluability assessment. Estados Unidos: Sage; 2015. 181 p.

15. Hartz ZMA, Vieira-da-Silva LM. Avaliação em saúde: dos modelos teóricos à prática na avaliação de programas e sistemas de saúde. Rio de Janeiro: Fiocruz; 2005. 275 p.

16. Leviton LC, Khan LK, Rog D, Dawkins N, Cotton D. Evaluability Assessment to Improve Public Health Policies, Programs, and Practices. Ann Rev Public Health 2010; 31: 213-33. https:// doi.org/10.1146/ annurev.publhealth.012809.103625

17. Baratieri T, Nicolloti C, Natal S, Lacerda JT. Aplicação do Estudo de Avaliabilidade na área da saúde: uma revisão integrativa. Saúde Debate 2019; 43(120): 24055. https: / / doi.org/10.1590/0103-1104201912018

Recebido em: 01/10/2020

Revisado em: 23/11/2020

Aceito em: 24/11/2020

Contribuição dos autores: todos os autores participaram da construção da pesquisa, de sua gestão e da análise dos dados. AFB, JTL, ACB, SS, YTT redigiram a primeira versão do manuscrito e sua versão final. Todos os demais autores revisaram criticamente o texto e contribuíram para a versão final. 